\title{
The Role of Ayod Community Health Teams in Promoting Maternal and Infant Health in a Municipality in Ifugao, Philippines
}

\author{
Floreliz V. Ngaya-an ${ }^{1}$ and Cathrine Fowler ${ }^{2}$ \\ ${ }^{1}$ College of Nursing, University of the Philippines Manila, Philippines \\ ${ }^{2}$ Tresillian Chair in Child and Family Health, University of Technology, Sydney, Australia
}

\begin{abstract}
Objective. This study investigates the role of the Ifugao Community Health Teams, or the Ayods that function as frontline health workers, responsible for tracking pregnant women, bringing them to clinics for check-ups and encouraging planned facility-based birth. Specifically, this study examines their contribution to the Maternal and Child Health $(\mathrm{MCH})$ Project, jointly initiated by the Philippines Department of Health (DOH) and Japan International Cooperation Agency (JICA). Commencing in 2006 and ending in 2010, the project aimed to improve the health and safety of mothers and newborns by ensuring the availability of quality health care, and increasing utilization of health facility and services.
\end{abstract}

Methods. A qualitative interpretive descriptive approach was used to guide the study. Three focus group discussions were conducted to collect data from a total of 21 participants, who were active members of the Community Health Teams in one municipality in Ifugao. To work with these data, an a priori template analysis was used to assist in identifying themes within the data.

Results. This study has highlighted three major themes: (1) Collaborative efforts of the Ayods play a role in the achievement of significant health outcomes for the community; (2) Equity, access, and beliefs are key factors in the delivery of health care; and (3) Sustainability is secured through self-reliance and volunteerism.

Conclusion. The engagement of Ayods to trace and support pregnant women appears to have several successful health outcomes. The employment of existing community health workers and volunteers enabled the creation of a sustainable model for isolated communities. It is anticipated that this vital service will continue to be provided. Nevertheless it will require ongoing and increasing support through the provision of

Corresponding author: Floreliz V. Ngaya-an, RN, MAN

College of Nursing

University of the Philippines Manila

WHO Collaborating Center for Leadership in Nursing Development

University of the Philippines Manila

Sotejo Hall, Pedro Gil Street

Ermita, Manila 1000 Philippines

Telephone: +6325231472

Telefax: +632 5231485

Email: lizngayaan@gmail.com allowances, education and community commitment to voluntary work.

Key Words: community health teams, facility-based births, pregnancy tracking, maternal and child health, antenatal care

\section{Introduction}

Maternal mortality rate has declined by $45 \%$ from 380 per 100,000 live births in 1990 to 210 in 2013. The annual decline rate of $2.6 \%$ is impressive, but this remains unable to meet the required rate of $5.5 \%$, necessary to achieve the Millenium Development Goal number 5. ${ }^{1}$ Philippine data reflects that 120 women died in every 100,000 live births. ${ }^{1}$ More than half of the births in the Philippines occur at home. One third of these home births were assisted by the traditional birth attendants. ${ }^{2}$ A major concern with nonfacility based births is lapses in aseptic technique due to inadequate infection control measures, and lack of availability of suitable equipment when needed for emergency situations. ${ }^{3,4}$

In 2006, the Philippine government, through the Department of Health, signed an agreement with the Japan International Cooperation Agency (JICA), to provide technical assistance to the Maternal and Child Health Project in Ifugao and Biliran. ${ }^{5}$ The project was envisioned to support targets under the Millennium Development Goals to reduce the ratio of maternal deaths and decrease the mortality rate among children. It was aimed at improving the health and safety of mothers and newborns by ensuring the availability of quality health care, and increasing utilization of health facility and services. ${ }^{5}$

Facilities were upgraded, and trainings were provided for the doctors, nurses and the midwives, as part of capacity building. ${ }^{5}$ Community Health Teams, or the Ayods, as they are called in Ifugao, were organized and trained. ${ }^{5}$ Their role is to track pregnant women and bring them to the hospital or clinics for prenatal check-up, and to encourage them to plan for facility-based birth. Ayod is a native Ifugao word which means 'hammock'-a traditional method for carrying ill individuals from their home in the mountain, down to where the hospital or clinics are located. Ayod now refers to the groups of community people, who function as frontline health workers, delivering health information to people in their homes. 
In low resource settings like the Philippines, many doctors and nurses have flocked to the cities, leaving remote communities understaffed and underserved. To fill the gaps, Community Health Teams are engaged to help in the delivery of health care services to the people. Not much is known about the practice of the Ayods in Ifugao, or that of the other Community Health Teams in the Country. Of interest is the acknowledgment of the role of the Ayods and how they contribute to health outcomes, specifically that of the mother and the infant, thus the need to conduct this study.

The organization of the community health teams commenced with simultaneous local meetings called by the barangay* captains. Organizers of the project came and talked about the plan to organize the teams, and explained their purpose, as well as responsibilities of future members. Midwives, barangay officials and barangay health workers attended the meetings. The barangay captains and midwives supervising the health workers and nutrition scholars were already appointed and they became obligatory members. Nutrition scholars are trained community health workers, specifically concerned with nutrition of children in the barangay. When the teams were organized, the following additional members were included: barangay captain as chairman, midwife as co-chairman, the health workers, the nutrition scholars, and other volunteers. After the first meeting, seminars and training sessions were provided in the barangays, and the municipality center. These lectures included information about the care of the pregnant woman, and the Ayods' roles and responsibilities.

This study was conducted in 2011, after the $\mathrm{MCH}$ Project of JICA in Ifugao officially ended in 2010. A careful process of acquiring permissions from the Provincial Health Office of Ifugao, and the Municipality of Mayoyao was secured. The Provincial Health Office of Ifugao was provided a copy of the study results.

\section{Methods}

To guide this study a qualitative interpretive descriptive approach $^{6}$ was used as it acknowledges the context-bound and contextual nature of human experience. ${ }^{7}$ This approach is not prescriptive, nor is there a process of a circumscribed sequence of steps to be followed. ${ }^{6}$ An interpretive descriptive approach aims to provide relevant data and insights to contribute to the development of practice decisions by exploring and describing human experiences. ${ }^{7}$

\section{Participants and Recruitment}

The researcher met with the Provincial Health Officer to obtain permission to conduct the study. The Municipality of Mayoyao was recommended, being one of the three project sites of JICA in Ifugao. The nurse and the midwives of
Mayoyao were consulted, and three barangays were identified as relevant to the inquiry: Chaya, Balangbang and Tulaad. Chaya is close to the center of the Municipality where there is a hospital, while Balangbang and Tulaad are remotely situated but having upgraded birthing stations. Community Health Teams from the identified barangays were then invited to participate in the study. The researcher met with them, and explained the study purpose and objectives. Participants who were included were those who came to the meeting, were available and were willing to participate. All participants provided verbal consent prior to the commencement of the focus group discussions.

There were a total of 21 participants: 6 from Chaya; 7 from Balangbang; and 8 from Tulaad. The participants were mostly community women who were volunteer health workers from the time the MCH Project started in 2006, until the time it ended in 2010, up to when this study was conducted. All of them were female, married, and had either elementary or high school education. Aside from the community women, the midwives and the nutrition scholars were also there.

\section{Data Collection}

The researcher facilitated the focus group discussions using a semi-structured interview conducted in Filipino, the national language of the Philippines. There were three separate group discussions. The inquiry focused on four main areas: history of Ayods, work of the Ayods, challenges encountered, and perceived sustainability of the work. The data were transcribed verbatim and de-identified. Selected quotes were translated from Filipino to English.

\section{Data Analysis}

The analysis commenced with a reading and re-reading of the transcripts. An a priori template analysis was used to work with the data to ensure the study aim was addressed. The template was constructed using the four areas identified to guide the data collection. An a priori template analysis allows flexibility, as the template can be changed or additional categories added. ${ }^{9}$ The template was altered to include additional themes or findings found within the data. ${ }^{9}$ A final process was used that identified three major themes. As there were several participants in each focus group, identification of individual voices is not feasible.

\section{Findings of the Study}

Three major themes are highlighted as a result of this study: (1) Collaborative efforts of the Ayods play a role in the achievement of significant health outcomes for the community; (2) Equity, access, and beliefs are key factors in the delivery of care; and (3) Sustainability is secured though self-reliance and volunteerism. 
(1) Collaborative efforts of the Ayods play a role in the achievement of significant health outcomes for the community

Motorized tricycles are a common means of passenger transport in the Philippines especially the rural areas, being used also to carry patients to the hospital or the clinics. Unfortunately, this form of transport has limited use or is not viable in areas where access is difficult due to very high and remote locations. In Ifugao, people use an ayod or a hammock to carry patients instead. The ayod required people to carry, thus the word 'inaayod,' which means 'being carried.' To the Ifugaos, it is helping each other. The meaning of ayod is now extended to refer to a group of people helping each other.

In 2006, the JICA organizers commenced work, organizing the Women's Health Teams. The groups were composed of mainly preexisting barangay health workers and nutrition scholars. In 2008, the name 'Women's Health Teams' was changed to Ayod Community Health Teams. Female members were often faced with the need to carry laboring women down to the clinics and the hospital. As one Ayod from Chaya explained, it became Ayod Community Health Teams to emphasize the words 'Ayod' and 'community'. This is due to the coming in of male members, in order that there will be men to carry the laboring woman.

The removal of the gender specific term of women potentially encouraged more men to be involved, as it reduced the concept of this role being delegated only to women.

The main activity of the Ayods is to track pregnant women in their barangay. The Ayods reported that they are doing 'zoning' as strategy to ensure that the barangay is totally covered, with each Ayod assigned to a specific area, to enable all pregnant women to be identified early. Once identified, the women are listed and the clinic midwives are notified.

When pregnant women are identified, the Ayods discuss with them the importance of receiving prompt antenatal care to ensure the health and welfare of the mother and baby. The Ayods inform the pregnant women of the dangers of giving birth at home where there is no skilled attendant, and no equipment is available in case of emergency. They encourage the women to plan for a facility-based birth or clinic birth. Among the things that they need to plan for include: a source of finances, baby's clothes and other needs, a companion during labor, and a person who will stay with the other children who will be left at home. The transportation of the woman is a key aspect that needs to be managed. This role has been delegated to the barangay captains. They are the ones who facilitate transportation for the laboring woman.

The Ayods also visit the home of pregnant women to inform them that there are free supplements at the clinic.
They continue to visit the mother in her home even after the birth of the baby, in order to convince her to return to the clinic for postnatal check-up and immunization of the baby. Ayods from Tulaad said that they do not do home visit as an official activity all the time, preferring alternative methods. Sometimes they meet them informally in gatherings like the Sunday mass, and during common conversations while working in the field. They think they tend to interrupt the mothers' performance of the household chores when they visit them at home. Whenever they meet the mothers on the street, they often ask them how they are doing.

This approach, while not part of the usual service delivery protocol, seem to be working both for the pregnant women and the Ayods. A more conversational approach potentially reduces tensions related to issues of authority and provides opportunities for questions to be asked by the women and their families.

The Ayods claim that more women are now coming for prenatal check-up. Those convinced to give birth in the clinic increased in number. This is supported by the project annual report wherein facility based deliveries in the project areas in Ifugao increased from 30\% in 2005 to $79 \%$ in $2007 .{ }^{5}$ Maternal and infant mortality also declined. As one Ayod from Tulaad reported, there are no more women dying because of profuse bleeding. According to the project annual report, maternal mortality ratio decreased from 85.2 in 2005 to 0 in 2007, infant mortality rate- from 8.5 to 10 , and neonatal mortality rate- from 5.1 to $5 .^{5}$

This reduction in mortality as described provides evidence of achieving a significant health outcome for the community in which this Ayod works.

As part of the role they play, the Ayods talk to women about breastfeeding and family planning. There are an increasing number of mothers who are breastfeeding now, and the pregnancy rate has decreased as a result of the campaign to enhance the use of contraceptives. The participants claimed that breastfeeding is better and fewer women are getting pregnant.

(2) Equity, access, and beliefs are key factors in the delivery of care

One major challenge that the Ayods encounter involves women who do not want to give birth in the clinics. While many women are now deciding to comply with the policy to give birth in a clinic, there are those who remain unconvinced. The reason for reluctance to birth away from home frequently relates to significant concern about the financial cost. Participants explained that pregnant women lack financial sources to pay for clinic births. An Ayod from Tulaad said, the mothers are telling them that they do not want to give birth in the hospital because they do not have money.

The participants pointed that while their services are free both in the barangay health stations and the rural health 
units, the hospital is charging around 2,000 to 3,000 Philippine pesos for a normal birth. PhilHealth membership is used as a means of assisting people pay for services. As most Ifugao people in Mayoyao are farmers, and only a few are teachers and government employees who receive a monthly cash salary, this results in many people being unable to afford membership to PhilHealth. In some circumstances, politicians and private individuals sponsor membership for the people. Only year-length memberships are available, with neither assurance of continued sponsorship nor assurance that new sponsors will be identified. Adding to this concern, the PhilHealth only pays for a maximum of four births resulting in pregnant women opting for homebirth for the fifth and subsequent pregnancies.

Some women are reluctant to attend the clinic for prenatal check-up or give birth in the clinic due to the distance from their homes as well as the condition of the roads. There are limited options for transportation. There are times in which women give birth on the way to the hospital.

Strong personal beliefs and practices influence the women's choice about using health services and giving birth in the hospital and clinics. One participant from Balangbang highlighted that there are mothers who are not convinced about the benefits of vaccination. One mother cited that her forefathers did not receive vaccinations and yet they did not get sick. The Ayod said the mother was angry because she felt that she is forced into something that she does not believe in.

The participant raised the delicate balance they needed to manage between promoting vaccinations and avoiding conflicts with personal beliefs. Participants identified that first-time mothers are usually easier to convince than older more experienced mothers to birth at a clinic. As an Ayod from Chaya illustrates: "Yes, the first timers... they are easy to convince. But mothers like us [participants referring to women their age and parity] they said 'I gave birth at the Kaingin field...nothing happened to me...so I will just give birth here [referring to home].'"

Some Ayods were conflicted, as they are required to encourage women to give birth in the clinic, informing them about the risks of homebirth. They confessed that they have a preference for home birth too. This is often an outcome of their experiences of successful births at home, either due to necessity or choice.

Midwives cannot be present at all times to handle birthing in the clinics, especially the unexpected births. This is often due to midwives having to attend to other responsibilities in other barangays. This results in pregnant women who initially decided to give birth in the clinicshaving a homebirth instead. The resulting outcome is a policy that prevents the provision of assistance during homebirths- being impossible to implement due to the urgency of the situation. As one participant from Chaya clarifies, sometimes they are obliged to assist in home births if the situation calls for it. When the mother is begging, they often cannot refuse.

The Ayods have personal needs that potentially impact their ability to perform their work. For example, they explained that they need umbrellas when they conduct home visits. If they do not have one, this may limit their ability to go out into the rain. This lack of basic equipment affects their ability to provide the necessary assistance.

The participants claim that supplies in the birthing stations are depleted and some equipment are no longer functioning. The fees for the services rendered are required to assist sustain each facility's operations. This is necessary in order to replenish the supplies, maintain the equipment, and the program as a whole. However, clinics, supplies and equipment are not well maintained due to the fact that fees are not collected in the health stations or the birthing clinics, as discussed earlier. Without fees there are no additional sources of money to provide or maintain a service to the required standard.

In addition to improvements in equipment and other health supplies, the Ayods identified the need to be adequately equipped as front line health workers to manage many of the health and other problems and concerns that arise for their communities. For example- taking BP and convincing people. Basic health procedures such as taking a blood pressure are identified as potentially enhancing the Ayods' role within communities. But the second skill identified- the need for enhanced communication skills, would enable the Ayods to become competent in convincing more women to give birth in the clinics and to advocate for their communities. Ongoing education is a necessary component of providing appropriate and safe health services to communities.

\section{(3) Sustainability is secured though self-reliance and volunteerism}

The Ayods' work is predominantly voluntary and their desire to help is not driven by money. As such, they believe the work will always be sustained, as long as there will be people who are willing to help.

The participants identified that the reward for participating in this voluntary work, is the positive feelings participants gain from helping others. The Ayods described their work as being very much part of their everyday life and a community culture of helping each other. The following quote from an Ayod from Balangbang highlights the commitment to the communities in which they live: "That is what we are actually doing already, and will continue to do... anybody can do it even if they are not an Ayod."

The quote provides a reinforcement of the Ayods' commitment to their communities. The quote also creates an 
impression that they do not position themselves as being any more skilled than other community members.

The Ayods even though they provide their services on a voluntary basis are paid an allowance, funded by their barangays as an incentive. These allowances differ from one barangay to another. The allowances come from the barangay's revenues, and additional funding is provided at the municipal level. An Ayod from Balangbang explained that the money the Barangay Health Workers receive, come from the barangay, $20 \%$ is provided by the Internal Revenue Allotment.

Local revenues are identified as a means of developing self-reliance and continuing support for community work and to encourage community members to take on more demanding roles.

\section{Discussion}

This study has demonstrated the relevance of the Ayods' work in Ifugao in the promotion of maternal and infant health. There were many positive outcomes for the women, the Ayods, and their community. Early pregnancy tracking, for example, is vital to encouraging more women to go to the clinics for prenatal check-up. The Ayods report that primipara women are now more likely to go for a prenatal check-up and give birth in the clinics. Prompt and appropriate care in the antenatal period is crucial to the health of the mother and the fetus, and to the outcomes of the pregnancy. ${ }^{10}$ Of note, are the Ayods' reports of a reduction in maternal and infant mortality, an increase in breastfeeding rates and a decrease in pregnancy rates. These outcome findings have been supported by the project annual report, wherein facility based deliveries in the project areas in Ifugao increased from $30 \%$ in 2005 to $79 \%$ in 2007.5 According to the same project annual report, maternal mortality ratio decreased from 85.2 in 2005 to 0 in 2007, infant mortality rate- from 8.5 to 10 , and neonatal mortality rate- from 5.1 to 5.5

The study findings demonstrate that the Ayod teams are working to reduce the factors, identified by the United Nations Population Fund (UNFPA) Report ${ }^{11}$ as contributing to the increase in the maternal mortality ratio (MMR): 1) delays in deciding to seek medical care; 2) delays in reaching appropriate care; and 3) delays in receiving appropriate care. The many issues the Ayods and the women in their community encounter when managing pregnancy and birth often impact all three factors and result in delays in receiving appropriate and safe medical care. For example, lack of access to services creates delays and impacts on all three of the above factors seeking medical attention, reaching and receiving appropriate care.

Accessibility is a major concern in areas where the provision of transportation is difficult and at times impossible. In research conducted in Yapacaní, Bolivia, it was revealed that distance from services or other geographic issues, were identified as a significant barrier (21\%) to timely access to health care services. ${ }^{11}$

While more and more women are now giving birth in clinics, there are those who still opt for home birth, despite attending antenatal clinics. For others clinic-based birth is not an option due to distance, a rapid labour and birth, and lack of finances or health insurance. A significant factor impacting on the decision to give birth in a clinic is the personal belief of the women. This creates tension for some of the Ayods, as their preference is also to give birth at home and not in a clinic. This may not be helpful for their campaign to promote clinic-based births, as the women may be looking to them as role models. If some of the Ayods do not want to give birth in the clinics, it is questionable that they will be able to convince other pregnant women to do so. Further research is required to explore this concern.

For some of the Ayods, a conflict is present as the government prohibits those who are not licensed from assisting in childbirth. The Ayods frequently encounter women still in their homes who need help at the time of births. Some of the Ayods were traditional birth attendants without formal education to manage births. Even though they have significant experiences in caring for women during labour and birth they are legally unable to provide the needed assistance during childbirth. The need for skilled attendance being vital for safe birthing is essential. But it cannot be denied that in the Philippines, there is scarcity of health resources including people resources as many health workers are attracted to working in other more wealthy countries. ${ }^{12,13}$ Midwives are not always available due to either distance, other women requiring their assistance at birth or other workload issues. Women in these situations have no option but to turn to the Ayods or other traditional birth attendants to assist them during labour and birth.

In Biliran (the other project site of JICA), penalties were imposed on traditional birth attendants attending home birth. ${ }^{5}$ This was in an attempt to support the campaign for clinic based-births and births with skilled attendance. This was made possible through the local government's active support of the campaign. This approach using penalties has been found to be effective. ${ }^{5}$

In Ifugao, no strict restrictions or penalties were used to support the campaign. This could be viewed as community leaders inclined to maintaining sensitivity to cultural beliefs and practices. While it is beneficial for women to be attended by skilled personnel during labour and birth, it is also equally important for an Ifugao woman to have somebody who is familiar, whom she could trust and feel comfortable with during childbirth. Not imposing penalties could be interpreted as a way to give people time for adjustment, for them to see the benefit, and be able to decide for themselves.

Organizations that provide help for communities should support culturally sensitivity processes. Although it may be ideal for traditional birth attendants to no longer 
attend births, this situation must be dealt with sensitively as it is a longstanding culture practice. The restriction on traditional birth attendants in many communities may be counterproductive, especially if there are insufficient numbers of midwives to support the women during labour and birth. Research into health workforce needs is required to identify the skill profile that is required in Philippine communities. Expansion of the health skill mix to include community nurses and other health workers may be an effective clinical and cost efficient approach.

Further research is required to identify and develop a best practice model of care during pregnancy, labour, childbirth and postnatal period. This model of care needs to be culturally appropriate to the Filipino communities. It needs to address the concerns of the women and scarcity of resources.

If pregnant women are required to give birth in clinics, PhilHealth membership especially for those who cannot afford contribution must be reviewed and strategies developed to ensure health insurance coverage. Fees are crucial to operate clinics, as supplies need to be replenished, and equipment needs to be maintained.

\section{Conclusion}

The use of Ayods to trace and support pregnant women appears to have had many successful health and other outcomes. The project has used existing community health workers and volunteers that have enabled the creation of a sustainable model for isolated communities. There remain some significant challenges that need to be addressed to ensure ongoing sustainability of this project.

Enhancements to the project are necessary to ensure that the best possible care is provided, and services remain responsive to the needs of the community, particularly through increased knowledge and skills development and updates to adequately support the Ayods and their developing knowledge and skills. This would enable them to be appropriately equipped to safely manage many of the unplanned and often-emergency situations they encounter.

There is good reason to believe sustainability of this vital service to the community will be continued. Nevertheless, it will require ongoing and increasing support through the provision of allowances, education and a commitment by the community to voluntary work.

\section{References}

1. World Health Organization, UNICEF, United Nations Population Fund and the World Bank. Trends in Maternal Mortality: 1990 to 2013. WHO [Online]. 2014 [cited March 2015]. Available from http://data.unicef.org/maternal-health/maternal-mortality.

2. United Nations Development Program. Millennium Development Goals: Improve maternal health. [Online]. 2014 [cited March 2015]. Available from http://www.ph.undp.org/content/philippines/en/home/ mdgoverview/overview/mdg5.

3. Ekanem A, Udoma E, Utsalo S. Bacterial contamination of women in labor supervised by traditional birth attendants. Int J Gynecol Obstet. 2006; 92(2):126-7.

4. Belton S, Myers B, Ngana F. Maternal deaths in eastern Indonesia: 20 years and still walking; an ethnographic study. BMC: Pregnancy \& Childbirth. 2014; 14:39. [Online]. 2014 [cited March 2015]. Available from http://www.biomedcentral.com/1471-2393/14/39.

5. Department of Health. Teaming Up for Safe Motherhood: The Biliran and Ifugao Experience. Republic of the Philippines Department of Health: Manila. 2008.

6. Thorne S. Interpretive description. CA: Left Coast Press. 2008.

7. Thorne S, Reimer Kirkhan S, O'Flynn-Magee K. The analytic challenge in interpretive description. International Journal of Qualitative Methods 2004; 3(1). [Online]. 2004 [Cited March 2015]. Available from http://www.ualberta.ca/ iiqm/backissues/3_1/pfd/thorneetal.pdf.

8. Lasiuk G, Comeau T, Newborn-Cook C. Unexpected: an interpretive description of parental traumas' associated with preterm birth. BMC: Pregnancy \& Childbirth. 2013; 13(suppl 1):5-13.

9. King N. Template analysis. In Symon G \& Cassell C (eds.), Qualitative methods and analysis in organizational research. London: Sage. 1998. pp. 118-134.

10. Sword W, Heaman M, Brooks S, et al. Women's and care providers' perspectives of quality prenatal care: a qualitative descriptive study. BMC Pregnancy \& Childbirth. 2012; 12:1-18. [Online]. 2012 [cited March 2015]. Available from http://www.biomedcentral.com/1471-2393/12/29.

11. Otis KE, Brett JA. Barriers to Hospital Births: Why do many bolivian women give birth at home? Rev Panam Salud Publica. 2008; 24(1):46-53. [Online]. 2008 [cited March 2015] Available from http://www.ncbi.nlm.nih.gov/pmc/articles/PMC4247708/.

12. Bach S. International migration of health workers: Labour and social issues, International Labour Office, Geneva. 2003.

13. Dawson A, Howes T, Gray N, Kennedy E. Human resources for health in maternal, neonatal and reproductive health at community level: a profile of the Republic of the Philippines, Human Resources for Health Knowledge Hub and Burnet Institute, Sydney, Australia. 2011. 